\title{
Effects of habituation on threat display and dominance establishment in the Siamese fighting fish, Betta splendens
}

\author{
JANICE A. MELISKA and CHARLES J. MELISKA \\ Monmouth College, Monmouth, Illinois 61462
}

\begin{abstract}
In three separate experiments, male Betta splendens were exposed, respectively, to mirror images, a variety of conspecifics, and to future opponents, prior to combat with unexposed subjects. Threat display to mirror images and to live opponents habituated significantly, but the outcomes of dominancesubordinance tests were not significantly affected by prior exposure to any of the threat-eliciting stimuli. Results are discussed in terms of stimulus and response specificity and possible chemical suppression of agonistic behavior.
\end{abstract}

Male Siamese fighting fish (Betta splendens) perform vigorous threat displays at the sight of other male conspecifics and even in response to their own mirror images (Johnson, 1972). It has also been frequently observed (Clayton \& Hinde, 1968; Figler, 1972; Peeke \& Peeke, 1970; Shapiro \& Schuckman, 1971) that this aggressive display wanes (habituates) with repeated exposure to the eliciting stimulus. Meliska (1974) reported that Bettas which had been visually exposed to each other and to their own mirror images, prior to engaging in combat, later required significantly less time to establish dominance-subordinance relationships than fish not so exposed. This led Meliska, Meliska, Hoyenga, Hoyenga, and Ward (1975) to hypothesize that central to the phenomenon of dominance establishment in Bettas was the process of habituation-a longlasting, suppressive effect on responding brought about by repeated exposure to a stimulus (Peeke \& Peeke, 1973).

According to this habituation hypothesis, fish whose aggressive responding habituates at a slow rate should dominate fish whose aggressive responding habituates more rapidly. If so, it should be possible to manipulate social status, experimentally, by exposing one fish to aggression-eliciting stimuli prior to combat while giving its future opponent no such exposure. Since the aggressive responses of the exposed fish would be expected to become habituated to some degree, the fish should be at an offensive disadvantage when placed with an animal which had not received such exposure. Therefore, the latter would come to dominate the former.

The following series of experiments was designed to test the predictions implied by the habituation hypothesis concerning social status in Siamese fighting fish.

\section{EXPERIMENT I}

Since exposure to a mirror image produces habitua-

Requests for reprints should be sent to Charles J. Meliska, Department of Psychology, Monmouth College, Monmouth, Illinols 61462 . tion of threat behavior, it was predicted that fish which had viewed their own mirror images for extended periods would fight less vigorously than unhabituated controls, and would therefore prove to be subordinate to non-mirror-exposed opponents.

\section{Method}

Subjects. Forty-six male Bettas, acquired from a local fish dealer, were assigned to 23 pairs. Pair members were matched for size and randomly assigned to either the Mirror or Unexposed groups. (Each pair included one mirror and one unexposed fish.) Animals were kept singly in 1-liter, translucent jars maintained at a water temperature of $25^{\circ} \pm 2^{\circ} \mathrm{C}$. A $14 \mathrm{~h}$ light 10-h dark cycle was in effect. Fish were fed freeze-dried brine shrimp once each morning.

Procedure. After the animals were adapted to the laboratory for 17 days, two mirrors, each measuring $5.2 \times 7.7 \mathrm{~cm}$, were positioned in the home jars of the mirror group, so that the fish could see their mirror images. Two nonreflective panels were placed into each home jar of the unexposed group. (These panels were identical to the mirror group's mirrors, but were reversed to show the flat gray back.) Subjects were thus exposed to mirrors or panels for 12 consecutive days of $14 \mathrm{~h}$ of light followed by $10 \mathrm{~h}$ of total darkness.

Following 12 stimulation periods, mirrors and panels were removed. Fish were given a 24 -h period without stimulation to allow dissipation of fatigue. Pairs were then tested for establishment of dominance-subordinance relationships by placing each pair in a 4-liter glass jar filled halfway with aged tap water. Observations were made for $1 \mathrm{~h}$ in a darkened room. The glass jar was illuminated by a $25-\mathrm{W}$ overhead lamp. The criterion for dominance establishment was met when one fish fled from the approach of the other for 20 consecutive times. If the criterion was not met during the 1-h period, fish were returned to their home jars. Pairs were then placed together again under the same conditions for $30 \mathrm{~min}$ on each succeeding day until the dominance criterion was met. Fresh aged tap water was used for each test.

\section{Results}

Bouts ranged in length from 5 to $165 \mathrm{~min}$, with a mean of $78 \mathrm{~min}$ required to establish dominance.

Of the 23 pairs, 13 of the unexposed fish emerged dominant, while 10 of the mirror fish were dominant. Though in the expected direction, this outcome was not significantly different from chance expectation 
$(p=.339$, binomial test, Runyon \& Haber, 1971, p. 310). Thus the prediction that unexposed fish would cominate mirror fish was not supported, suggesting that rnirror exposure did not significantly reduce the combativeness of Siamese fighting fish.

\section{EXPERIMENT II}

The results of Experiment I suggested that perhaps mirror images were too dissimilar from live opponents to provide for generalization of habituation of aggressive responses from the preexposure to the combat situation. |Gallup (1968), for example, has discussed the numerous important differences between mirror stimuli and the stimuli provided by live animals.] The second experiment was essentially a replication of the first, except that live fish were substituted for mirror images.

\section{Method}

Subjects. Thuty male Siamese fighting fish from a local tish dealer served as subjects. Animals were matched for size, assigned to pairs, and pair members were randomly assigned to ether the Live or Unexposed groups. Live group subjects were housed individually in square 1.2-liter glass jars. Unexposed group animals were housed individually in 1 -liter translucent Jars. All other conditions were the same as Experiment I except that animals received no exposure to mirrors or panels.

Procedure. After the live group subjects had been visually isolated from one another for $24 \mathrm{~h}$, their glass jars were placed side by side, allowing the animals visual access to one another. The position of each home jar was changed every 2 days so rach fish was exposed to all the other animals in the live group Subjects received such visual stımulation from live conspecifics tor 26 consecutive 14-h light periods, each followed by $10 \mathrm{~h}$ of darkness. Subjects in the unexposed group received no special treatment but merely remained visually isolated from one another in their home jars.

Following the 26th period of visual stimulation. Live group anımals were visually isolated from one another for $24 \mathrm{~h}$. Pairs were then tested for dominance as in Experiment $I$.

\section{Results}

Bouts ranged in length from 22 to $190 \mathrm{~min}$, with a mean of $95 \mathrm{~min}$.

Eight of the Live group fish dominated their opponents, while seven of the Unexposed fish were dominant. This small difference, in the opposite direction from the one predicted, was not significantly different from chance expectancy $(p=.5$, binomial test). Thus, prolonged viewing of a variety of live conspecifics did not appreciably reduce combativeness; results obtained using live fish to bring about habituation were not substantrally different from those obtained with mirror stimulation.

\section{EXPERIMENT III}

Results of the first two experiments suggested that the habituation of aggressive responding to a variety of conspecifics, or to one's own mirror image, did not effectively generalize to an unfamiliar opponent. There- fore, Experiment III was designed as a modification and refinement of the first two. Instead of merely exposing fish to a variety of other conspecifics, the animals in the live group were exposed only to their intended future opponents, via a one-way mirror. It was anticipated that the habituation of aggressive responding to a particular fish would generalize more readily to the combat situation if the animal the subject was exposed to was the same as the animal to be fought. Peeke and Veno (1973) reported that the habituation of sticklebacks' aggressive behavior generalizes, to some extent, from one fish stimulus to the same stimulus presented in a different location.

Additionally, in order to test whether habituation of the threat display was in fact resulting from the exposure period, both groups of subjects were observed for gill cover erection (GCE), to either a mirror image or to a subject responding to a mirror image, on the first and last days of the habituation period. GCE is used as an objective measure of threat display in the Siamese fighting fish (Adler \& Hogan, 1963; Figler, 1972; Simpson, 1968) and it was expected that GCE duration would diminish markedly due to exposure to both a mirror image and to the image of the opponent.

\section{Method}

Subjects. Twenty-four male Bettas were matched for size, assigned to pairs, and randomly assigned to either the live or mirror groups as described above. All animals were housed individually in square, 1.2-liter glass jars.

Procedure. After the fish had been adapted to the laboratory envoronment for seven days, each subject was observed for GCE to elther its own mirror image or to its opponent. The home jars of pair members were placed on opposite sides of a one-way mirror. Overhead illumination was raised on one side of the mirror so that the Mirror group was exposed to its own mirror reflection. The matched Live group subject was placed immediately opposite so that it could see through the glass to its Mirror group subject displaying to the mirror image. At no time could the Mirror group subject see its pair member through the glass. Mirror fish were seen against an illuminated white background.

A subject pair was observed simultaneously by two observers for GCE to either the mirror image or to the live fish. GCE was observed for $5 \mathrm{~min}$, preceded by a 10 -min adaptation period. The observers alternated observations between mirror and live group fish. A pair was removed from its position at the one-way mirror following GCE testing.

On the next day, all pairs were repositioned at the one-way mirror. Murror group subjects were placed adjacent to the mirror, while Live group subjects were placed on the opposite, transparent side. In each case, a fish could see only its own mirror image or only its conspecific. Animals were maintained in this situation for 12 cycles of $14 \mathrm{~h}$ of light followed by $10 \mathrm{~h}$ of dark. At the end of the 12 th light cycle, pairs were removed from the one-way mirror.

On the following day, subject pairs were again observed for GCE according to the same procedure used prior to any mirror or conspecific exposure. Dominance tests were begun on the day after GCE lesting. The dominance procedure was similar to the one used in Experiments I and II. However, animals were observed under the same illumination that Live group fish had experienced when positioned at the one-way mirror. 
Results

Figure 1 presents the mean number of seconds spent in GCE by both groups before and after exposure. GCE scores were analyzed by a $2 \times 2$ (Mirror vs. Live Group by Preexposure vs. Postexposure) factorial analysis of variance. Only the time factor was significant $(\mathrm{F}=19.7, \mathrm{df}=1 / 22, \mathrm{p}<.001)$, indicating that exposure to mirrors and to live conspecifics produced a significant decrement in threat display duration in both groups.

As for the dominance data, bouts ranged in length from 12 to $158 \mathrm{~min}$, with a mean of $79 \mathrm{~min}$.

Seven Mirror fish were dominant and five Live group fish were dominant-a nonsignificant difference between groups $(p=.387$, binomial test). Thus, visual exposure to a particular opponent, prior to combat, did not significantly reduce a fish's chances of becoming dominant over that opponent.

\section{DISCUSSION}

The results of Experiment III confirmed the expectation that prolonged exposure to the image of a conspecific or mirror would markedly reduce threat display duration. Indeed, by the end of the 12-day habituation period, fish were minimally reactive to either live conspecifics or mirrors, as shown by Figure 1. Nevertheless, fish habituated to live conspecifics were no more likely to be subordinate to their opponents than unhabituated fish. Thus, the results of all three experiments fail to support the prediction that the habituation of aggressive display via visual stimulation would make a fish more likely to become socially subordinate to an unhabituated animal. This outcome suggests either that the habituation of aggressive responding does not influence dominance establishment or that some other important factors were operating in these particular experiments. A few major possibilities are discussed.

One possibility has to do with response specificity. Since the threatening gestures made by physically separated fish represent only fractional, preliminary components of their total fighting behavior, it is logical that their habituation would diminish only a part of an animal's aggressive sequence. To the extent that actual attack behaviors like biting, butting, and fin tearing remain unhabituated, a fish's fighting behavior could remain quite vigorous in spite of habituation of the preliminary threat components. However, Figler and Klein (1974) found that the actual attack behaviors of male Siamese fighting fish were significantly decreased by prior exposure to one another for $6 \mathrm{~h}$. Thus, the habituation of appetitive components reduced the consummatory components of a Betta's sequential aggressive behavior. It is noteworthy also that Meliska et al. (1975) observed a strong relationship between the vigor of threat displays and dominance in Bettas; fish which threat-displayed more than their opponents in

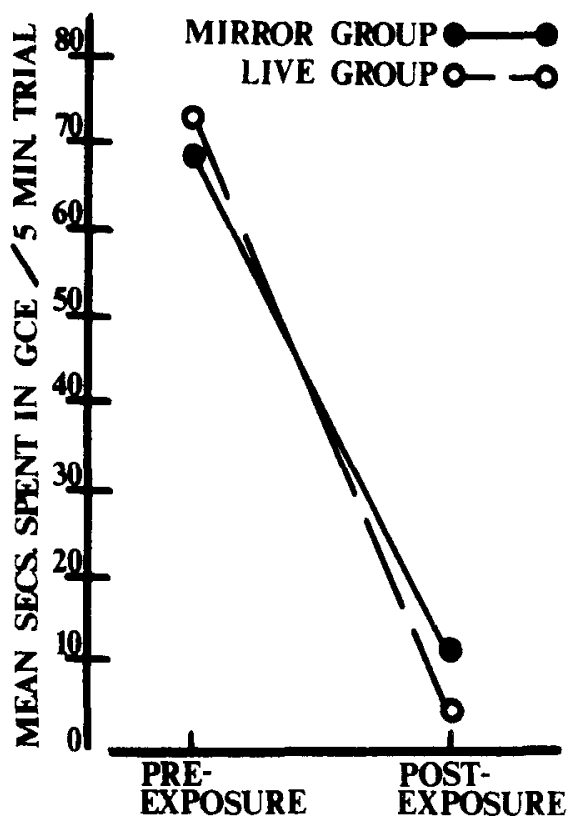

Figure 1. Mean seconds spent in gill cover erection by the mirror group and the live group per 5-min trial both before and after stimulus exposure.

tests prior to combat later dominated those opponents in about $90 \%$ of the cases tested.

Another possible explanation of the current findings has to do with stimulus specificity. It may be the case that the habituation of a particular response is limited to a specific stimulus or set of stimuli and does not generalize readily to other stimuli. Peeke and Peeke (1973) have discussed stimulus specificity in terms of a territorial fish being able to discriminate a neighboring animal from an intruder. The basis for such discrimination may be either individual cues characteristic of a conspecific or situation-geographic cues specific to a place or situation. Aggressive responding may habituate only to a particular individual, or to an individual seen in a certain context. In Experiment III, subjects in the Live group were exposed to their future opponent and threat display did decrease significantly. In five cases, however, these same subjects were still able to generate the appropriate aggressive behaviors and dominate their opponents. This could be due to the fact that the fish seen in the habituation trials was not responded to as the same stimulus seen during dominance testing. It is possible that the change in physical setting from habituation trials to dominance testing was responsible for the change seen in behavior. For example, the shape of the containers was different, as was the volume and freshness of the water. Peeke and Veno (1973) report that the generalization of habituation seen in the aggressive behavior of sticklebacks depends on two factors: the test stimulus should be similar to the stimulus presented during the habituation 
trials and the location of stimulus presentation should be similar to the location used for the habituation trals.

Another change in stimulus conditions from the habituation period to the dominance test sessions may be ascribed to changes in context. When the Live group subjects viewed their opponents through the one-way rnirror. the fish pairs were separated by boundaries enforced by the intervening glass. In later dominance houts, the fish were free to physically interact. Baerends and Baerends-von Roon (1950) point out that boundary fights between resident cichlids differ from intraterritorial fights between resident and intruder. Since the two types of fighting differ in form, it is likely that the amount of generalization of inabituation between the two types of aggressive encounters would also be less (Peeke \& Peeke, 1973). The inequality of boundary and intraterritorial contexts may also be true for the behavior of the Siamese fighting fish.

The habituation of aggressive behavior may be an extraordinarily stimulus-specific process. The high degree to which it is specific is suggested by Baenninger and Mattleman (1973). Male Bettas were presented with two mirrors-one continually present and the other contingent upon an operant response. Habituation of the aggressive display occurred to the free mirror while aggressive display was increasing to the contingent mirror. In other words, virtually no generalization of habituation occurred between the similar stimuli provided by the free and contingent mirrors.

So far, only visual stimulation has been discussed as the mode for habituation, but the role of chemical stimulation may be important as well. Bardach and Todd (1970) emphasized the importance of pheromones for communication in nocturnal fish and those residing in turbid waters. The domesticated Siamese fighting fish, used widely for research purposes, has been derived from the wild variety found in the muddy, sluggish waters of Thailand (Gordon \& Axelrod, 1968). Under these conditions, the development of chemical senses would assist in the regulation of behavior should visibility be low.

Furthermore, it has been reported that a Betta's threat display to a mirror image is reduced by exposure to water once occupied by male combatants, by conspecifics of either sex, or by pieces of torn fin (Baenninger. 1968; Ingersoll, Bronstein, \& Bonventre, 1975). These results imply that water-borne chemicals influence the aggressive display of the Siamese fighting fish, producing attenuation of display similar to that produced by visual access to a mirror image or conspecific. Hence, habituation of agonistic responding may be the product of both chemical and visual influences.

The composition of the chemical environment produced by a fish represents another variable that may account for the present experiments' results. Subjects were maintained in their home containers during exposure to a mirror image or to $\mathrm{i}$ conspecific. Fish were later removed from their home water for dominance bouts. The chemicals excreted by the fish during their exposure to aggression eliciting stimuli may be part of the habituation mechanism. These chemical traces may represent a source of negative feedback in suppressing display. Removing the fish from its self-produced chemical environment may have produced dishabituation to some degree. Perhaps, if the Live group subjects had been tested for dominance in water taken from their home jars, the chemical effect plus the prior experience of exposure to the opponent would cause them to be subordinate to the mirror subjects. The effect of home jar water alone, apart from the effect of the home jar itself, on dominance in Siamese fighting fish has not been explored. Of course, such a relationship would have to be investigated before the combined influences of water-borne chemical traces and visual exposure to an opponent could be related to dominance establishment.

In summary, the present experiments suggest that the habituation of aggressive responding to visual stimulation arising from mirror images or from live conspecifics is not sufficient to reduce the agonistic behavior of fish when they are put into actual physical combat. This may imply that the process of habituation is relatively unimportant in dominance establishment, or that habituation of responding may be so specific that it does not generalize from one situation to another. Another possibility is that chemical changes in the water of combatants esther alone or in combination with visual stimuli-may provide the basis for habituation of aggressive responding and dominance establishment.

\section{REFERENCES}

Adler, N., \& Hogan, J. A. Classical conditioning and punshment of an instinctive response in Betta splendens. Animal Behaviour, 1963,11,351-354.

Baenninger, R. Fighting by Betta splendens. Effects on aggressive displaying by conspecifics. Psychonomic Science, 1968, slve displayin
$10,185-186$.

Baenninger, R., \& Mattleman, R. A. Visual reinforcement: Operant acquisition in the presence of a free mirror. Animal Learning \& Behavior, 1973, 1, 302-306.

Baerends, G. P., \& Baerends-von Roon, J. M. An introduction to the study of the ethology of cichlid fishes. Behaviour, 1950, Supp. 1,1-242.

Bardach, J. E., \& Todd, J. H. Chemical communication in fish. In J. W. Johnston, Jr., D. G. Moulton, \& A. Turk (Eds.), Communication by chemical signals (Vol. 1). New York: Appleton-Century-Crofts, 1970.

Clayton, F. L., \& Hinde, R. A. The habituation and recovery of aggressive display in Betta splendens. Behaviour, 1968, 30, 96-106.

Figler, $M$. H. The relation between eliciting stimulus strength and habituation of the threat display in male Siamese fighting fish. Behaviour, 1972, 42, 63-96.

Figler, M. H., \& Klein, R. M. Habituation of the appetitive components attenuates subsequent consummatory components of the agonistic sequence in male Siamese fighting fish (Betta splendens). Paper presented at the meeting of the Animal Behavor Society, Urbana-Champaign, Illinois, May 1974.

Gallup, G. G. Mirror image stimulation. Psychological Bulletin, $1968,70,782-793$. 
Gordon, M., \& Axelrod, H. R. Siamese fighting fish. New Jersey: TFH Publications, 1968 .

Ingersoll, D. W., Bronstein, P. M., \& Bonventre, J. The chemical modulation of agonistic display in Betta splendens (Pisces, Belontiidae). Journal of Comparative and Physiological Psychology, 1975. In press

Johnson, R. N. Aggression in man and animals. Philadelphra: Saunders, 1972.

Meliska, J. A. The effects of social experience and familiarity on the approach tendency and aggressive display of the Siamese fighting fish, Betta splendens. Paper presented at the meeting of the Animal Behavior Society, Urbana-Champaign, Illinois, May 1974

Meliska J A Meliska, C J Hoyenga, K , T Hoyenga, K. B. \& Ward, E. F. Approach tendency and threat display as related to social status of Siamese fighting fish, Betta splendens. Animal Learning \& Behavior, 1975, 3, 135-139.

Peeke, H. V. S., \& Peeke, S. C. Habituation of conspecific aggression responses in the Siamese fighting fish. Behaviour, $1970,36,232-245$.
Peeke, H. V. S., \& Peeke, S. C. Habituation in fish with special reference to intraspecific aggressive behavior. In $H . V$. $S$. Peeke \& M. J. Herz (Eds.), Habituation I: Behavioral studies. New Y ork: Academic Press, 1973.

Peeke, H. V. S., \& Veno, A. Stimulus specificity of habituated aggression in three-spined sticklebacks (Gasterosteus aculeatus). Behavioral Biology, 1973, 8, 427-432.

Runyon, R. P.. \& Haber, A. Fundamentals of behavioral statistics. Reading, Massachusetts: Addison-Wesley, 1971.

Shapiro, S., \& Schuckman, H. Habituation and covariation of the components of the threat display in Betta splendens. Psychological Reports, 1971, 28, 827-837.

Simpson, M. J. A. The display of the Siamese fighting fish, Betta splendens. Animal Behaviour Monographs, 1968, 1, 1-73.

(Received for publication August 1, 1975; accepted October 21,1975 .) 